\title{
Quasi-barrierless submolecular motion in mechanically interlocked carbon nanotubes
}

Julia Villalva, Belén Nieto-Ortega, Manuel Melle-Franco* and Emilio M. Pérez.*

\begin{abstract}
The motion of molecular fragments in close contact with atomically flat surfaces is still not fully understood. Does a more favourable interaction imply a larger barrier towards motion even if there are no obvious minima? Here, we use mechanically interlocked rotaxane-type derivatives of SWNTs (MINTs) featuring four different types of macrocycles with significantly different affinities for the SWNT thread as models to study this problem. Using molecular dynamics, we find that there is no direct correlation between the interaction energy of the macrocycle with the SWNT and its ability to move along or around it. Density functional tight-binding calculations reveal small $(<2.5 \mathrm{Kcal} \cdot \mathrm{mol}-1)$ activation barriers, the height of which correlates with the commensurability of the aromatic moieties in the macrocycle with the SWNT. Our results show that macrocycles in MINTs rotate and translate freely around and along SWNTs at room temperature, with an energetic cost lower than the rotation around the $\mathrm{C}-\mathrm{C}$ bond in ethane.
\end{abstract}

Understanding and controlling (sub)molecular scale motion remains a major challenge, yet it is an essential requirement for the development of viable mechanical and electronic nanodevices. ${ }^{1}$ One of the most attractive candidates to study long-range submolecular motion are mechanically interlocked molecules (MIMs), ${ }^{2}$ and, in particular, rotaxanes. ${ }^{3}$ In rotaxanes, one (or more) macrocycles are trapped into a linear compound (thread) by bulky substituents at its ends. The thread serves as a rail along and around which the macrocycles can move. Molecular shuttles are rotaxanes in which the macrocycle(s) are translocated from one binding site ('station') on the thread to a second station in response to external stimuli (for example: chemical fuels, light, $\mathrm{pH}$ or temperature) that invert the initial relative affinity of each of the sites for the macrocycle. ${ }^{3}$ The mechanism of motion is well understood: the macrocycle explores the full linear energy profile along the thread through Brownian motion, since there are two very well defined minima at each station, it spends most of its time at the deepest minimum, with the 
relative population at each station following a Boltzmann distribution (Figure 1a). ${ }^{4}$ Some of the most advanced synthetic molecular machines described to date are based on this molecular shuttle concept. ${ }^{5-12}$ In contrast, the motion of molecular-sized fragments with respect to one another along energy profiles with atomic-scale roughness, is still not fully understood. ${ }^{13-22}$ In a landmark study, Berman, Drummond and Israelachvili, studied friction between atomically flat non-adhesive mica surfaces, and concluded that friction force is proportional to the perpendicular load, but the atomic area of contact is not. ${ }^{23}$

Our group recently described the synthesis of this mechanically interlocked carbon nanotubes (MINTs) by encapsulation of single-walled carbon nanotubes (SWNTs) within organic macrocycles, to form rotaxane-like species. ${ }^{24-31}$ The translocation of organic dyes, ${ }^{32}$ "bulk" water, ${ }^{33}$ fullerenes, ${ }^{34,35}$ or individual DNA strands ${ }^{36}$ inside SWNTs have been studied before. In this context, MINTs are ideal model systems to study friction at the submolecular level. The perpendicular load on the macrocycle towards the SWNTS is naught, as the system is in equilibrium. Moreover, there are no clearly defined energy minima for the macrocycle along the SWNT, so we expect a fundamentally flat energy landscape for motion (Figure 1b). Interesting questions arise: does submolecular motion depend on the affinity of the macrocycle for the SWNT? In turn, is affinity univocally related to the area of short intermolecular contacts?

Here, we present a comprehensive study of the dynamic properties of MINTs. We use molecular dynamics to describe how shuttling (movement along the SWNT) and pirouetting (movement around the SWNT) motions depend on the macrocycle affinity towards the SWNTs and temperature. To get further insight, we use Density Functional Tight Binding (DFTB) calculations to estimate energy barriers for motion. 
a)

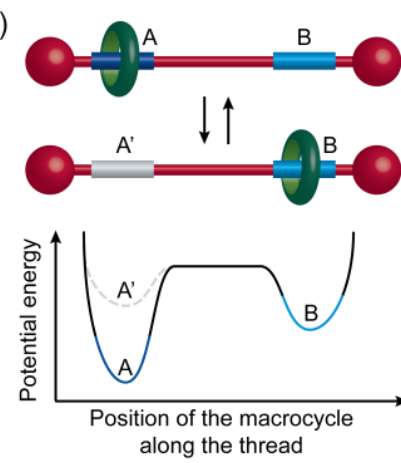

b)

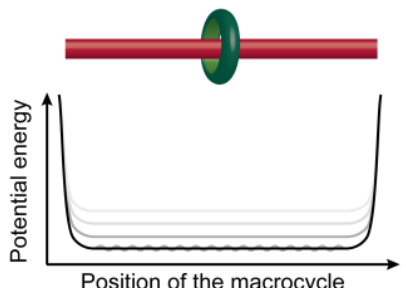

Position of the macrocycle

along the thread

Movement?

Figure 1. Schematic potential energy profiles for the movement of an imaginary macrocycle in a) a typical two-station molecular shuttle structure and b) a carbon nanotube (MINT model).

To better understand the submolecular motion in MINTs, we analyzed the relative movement between four different already described macrocycles -based on pyrene (Pyr) ${ }^{27} \pi$-extended tetrathiafulvalene (exTTF), ${ }^{26,28}$ anthraquinone (AQ), ${ }^{31}$ and naphthalenetetracarboxylic diimide (NDI) ${ }^{29}$ and $(6,5)-$ SWNTs (see Figure 2). This variety of MINTs allows us to elucidate if submolecular motion is related to the different interaction energies between the macrocycles and the carbon nanotube. In order to avoid geometrical effects due to different size of the macrocycles, the length of the aliphatic chain was chosen to ensure a similar diameter for all studied systems.

The interaction energy $\left(\mathrm{E}_{\text {int }}\right)$ between the macrocycle and the carbon nanotube was defined as the energy difference between the MINT and the individual moieties separately (nanotube and macrocycle), with all of them at the geometry of the final MINT. This quantity was calculated under the density functional theory (DFT) framework (see ref. ${ }^{31}$ and SI, S1 for details). The highest $\mathrm{E}_{\text {int }}$ at $-109.86 \mathrm{Kcal} \cdot \mathrm{mol}^{-1}$ was

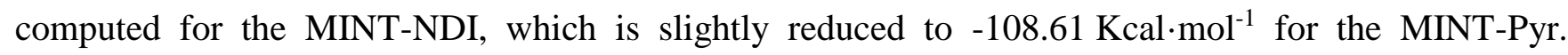
Moving to the anthracene-like macrocycles, the $\mathrm{E}_{\mathrm{int}}$ was computed at $-103.58 \mathrm{Kcal} \cdot \mathrm{mol}^{-1}$ for the MINTexTTF and $-97.74 \mathrm{Kcal} \cdot \mathrm{mol}^{-1}$ for the MINT-AQ. 
a)
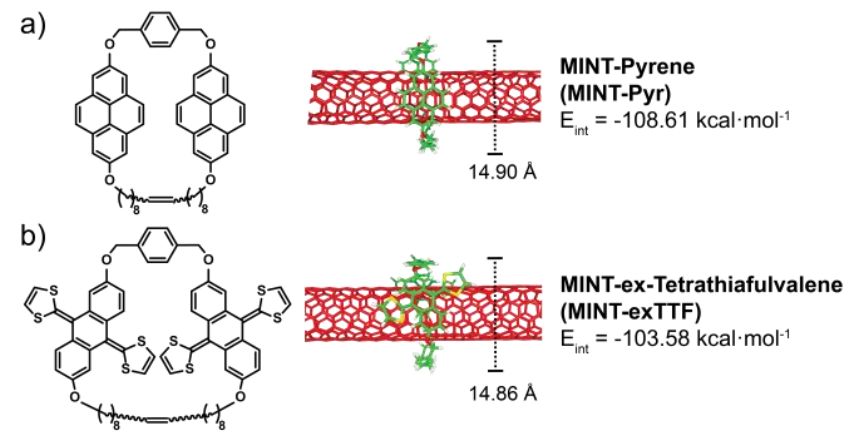

.
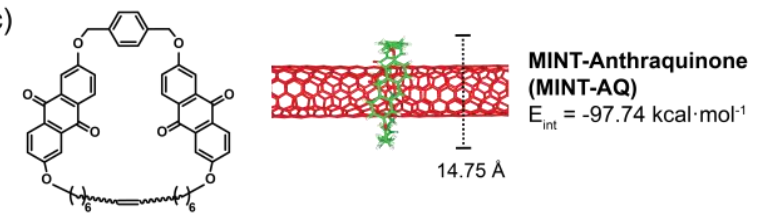

d)
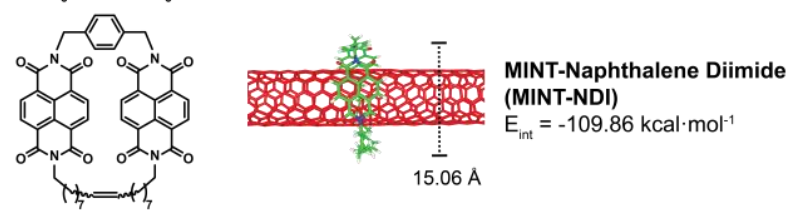

Figure 2. a) Chemical structure of a) pyrene-, b) exTTF- c) AQ- and d) NDI- based macrocycles and their corresponding optimized MINT structures.

Following the MIM nomenclature, two main kinds of large amplitude motion are defined: pirouetting, in which the macrocycle rotates around the tube, and shuttling, in which the macrocycle moves along it. In Figure 3, we followed the movement of the MINT-Pyr macrocycle along the SWNT during the molecular dynamics simulation at $300 \mathrm{~K}$. The shuttling movement is defined by the position of the macrocycle's centre of mass in each frame, starting with the macrocycle in the centre of the nanotube. For an easier representation, the initial position has been set in all trajectories as position zero (Figure 3a). The amplitude of this movement (difference between maximum distance in one direction (positive values) and in the opposite direction (negative values)) is used to measure the magnitude of shuttling. In the case of pyrene, the amplitude for the shuttling movement at $300 \mathrm{~K}$ is $54.9 \AA$.

In Figure $3 \mathrm{~b}$ the pirouetting movement is represented as the dihedral angle formed by one of the macrocycle atoms, a dummy atom inserted in the middle of the nanotubes and two dummy atoms located at the end of the nanotube. The initial angle is subtracted from the final data and the results obtained correspond to the degrees that the macrocycle rotates with respect to the initial orientation (see SI, S2 for details). The total pirouetting has been defined as the number of complete rotations that the 
macrocycle performs along the calculation. At $300 \mathrm{~K}$, the total pirouetting corresponds to 1.56 rotations/5 ns around the nanotube.

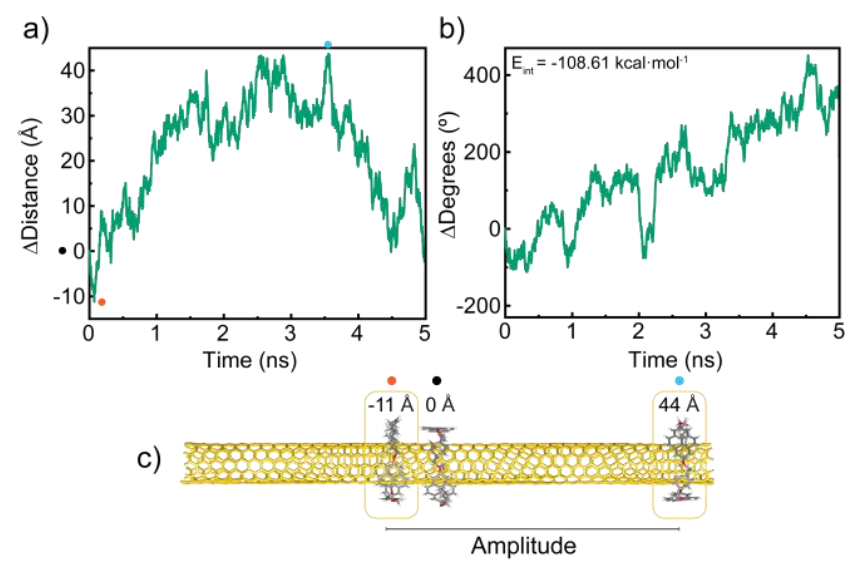

Figure 3. MD simulations for MINT-Pyr at $300 \mathrm{~K}$ showing a) translational movement and b) pirouetting movement. c) Overlapping images obtained from the MD simulation for MINT-Pyr showing the zero position ( $0 \AA$, black circle), maximum distance in the positive direction $(44 \AA$, blue circle) and maximum distance in the negative direction $(-11 \AA$, orange circle). The three different situations are marked with the same colour code in a).

The same trajectories were studied for MINT-exTTF, MINT-AQ and MINT-NDI, which present different aromatic moieties and consequently interaction energies. In Figure 4, the shuttling amplitude and number of complete rotations are presented for each MINT (see SI, S3 for detailed trajectories). All systems move freely along the nanotube, covering more than half of the SWNT's length (98.2 ̊). Moreover, movement is not dependant on the interaction energy between macrocycle and SWNT. MINTs with higher $\pi-\pi$ contact -NDI and -Pyr show larger amplitudes while the number of complete rotations varies between systems, being MINT-Pyr and MINT-AQ the ones that rotate more.
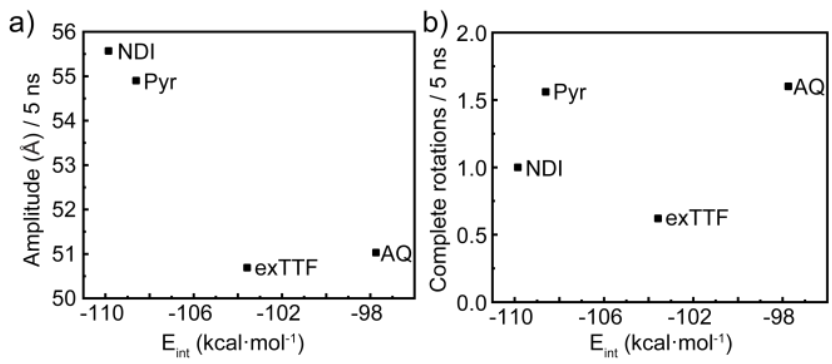

Figure 4. a) Total amplitude and b) complete rotations at $300 \mathrm{~K}$ for the studied MINTs. 
MD simulations at temperatures ranging from $1 \mathrm{~K}$, as the virtually static reference, to $500 \mathrm{~K}$ for MINT-

Pyr are presented in Figure 5. At $100 \mathrm{~K}$, the macrocycle's energy is greater than the activation energy for shuttling and pirouetting and the macrocycle starts moving. Rising the temperature increases the rate at which the macrocycle moves, which is reflected in higher amplitudes and rotation.
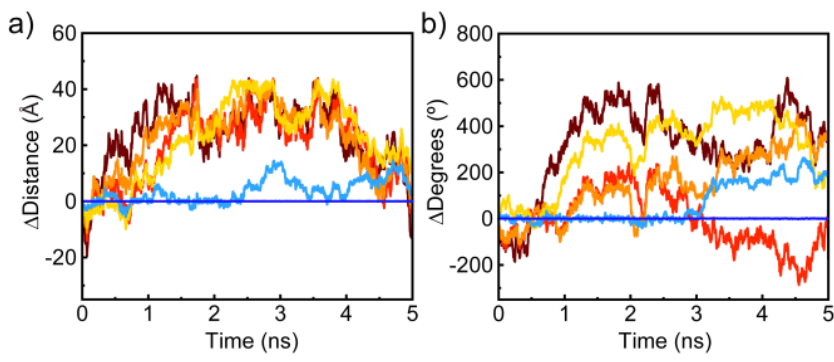

Figure 5. MD simulations for MINT-Pyr showing a) translational and b) pirouetting movements at different temperatures: $1 \mathrm{~K}$ (dark blue), $100 \mathrm{~K}$ (pale blue) $200 \mathrm{~K}$ (yellow), $300 \mathrm{~K}$ (orange), $400 \mathrm{~K}$ (red) and $500 \mathrm{~K}$ (dark red).

The same behaviour is observed for the rest of the MINTs studied (see SI, S4 and S5). In Figure 6, the shuttling and pirouetting is presented for the different MINTs and temperatures as a function of the $\mathrm{E}_{\text {int. }}$ For pirouetting, the number of complete rotations has been converted to actual displacement and again, the total amplitude of this movement has been plotted. Moreover, the sum of both pirouetting and shuttling is presented as total movement. As for MINT-Pyr, at $1 \mathrm{~K}$ there is no displacement, while at $100 \mathrm{~K}$ all systems start moving. Interestingly, for MINT-NDI and MINT-exTTF, higher temperatures are needed for the systems to initiate shuttling, indicating higher activation energies. Once the systems start moving, higher temperature implies larger displacements in form of shuttling and pirouetting. An interesting fact of MINTs movement is that the magnitudes of shuttling and pirouetting are comparable, indicating that they are controlled by similar energetic barriers. Finally, as observed for the $300 \mathrm{~K}$ case, the total movement does not depend on the affinity between macrocycle and SWNT. 


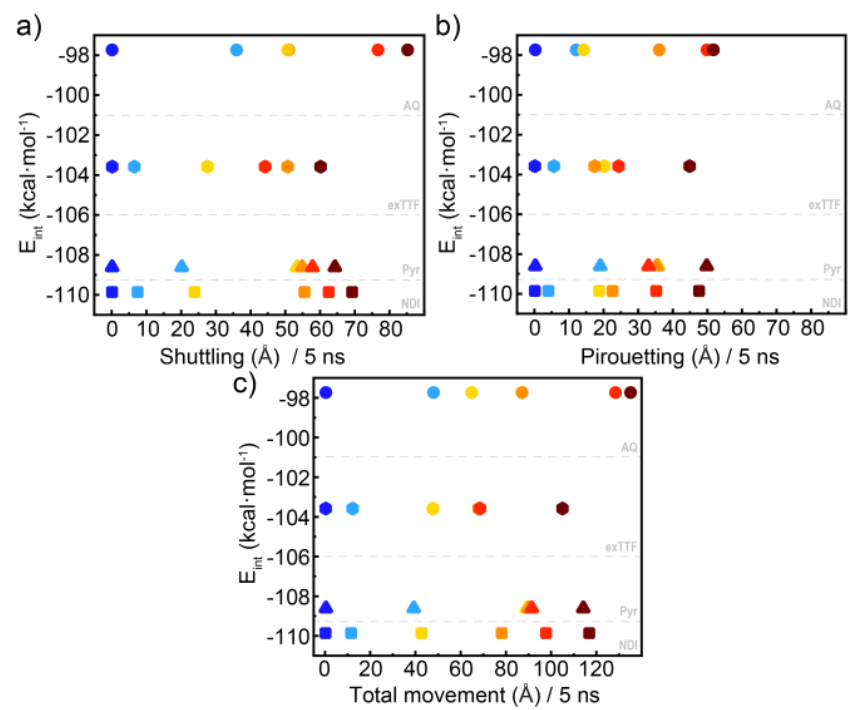

Figure 6. a) Total amplitude for shuttling and b) total amplitude for pirouetting presented as a function of the interaction energy for the four different MINTs; MINT-AQ (circles), MINT-exTTF (hexagons), MINT-Pyr (triangles) and MINT-NDI (squares) at different temperatures: $1 \mathrm{~K}$ (dark blue) $100 \mathrm{~K}$ (pale blue), $200 \mathrm{~K}$ (yellow), $300 \mathrm{~K}$ (orange), $400 \mathrm{~K}$ (red) and $500 \mathrm{~K}$ (dark red). c) Total movement performed for each of the four macrocycles at the six different temperatures. In a), for MINT-AQ, the values for $200 \mathrm{~K}$ and $300 \mathrm{~K}$ (yellow and orange circles, correspondingly) are overlapped. In c), for MINT-exTTF, the values for $300 \mathrm{~K}$ and $400 \mathrm{~K}$ (orange and red circles, correspondingly) are overlapped.

The data obtained employing molecular dynamics point to a quasi-barrierless molecular motion, in agreement with zero-load, flat energy profile, like that shown in Figure 1.

To get an atomic level understanding of the energetics behind this behaviour, we performed semiempirical calculations at the DFTB-D3 level for the displacement of the macrocycles. These models allow to efficiently access quantum-based models on systems with thousands of atoms. ${ }^{37}$ The activation barrier was estimated by monitoring the change of energy due to the displacement of each macrocycle parallel to the SWNT axis. A maximum was found in all cases; at 1.25 and $1.20 \AA$ for MINT-NDI and MINT-Pyr and 1.40 and $1.20 \AA$ for MINT-AQ and MINT-exTTF respectively. A minimum was found also for MINT-NDI, MINT-Pyr, and MINT-AQ at near the armchair periodic distance. This indicates that the chosen SWNT section is representative, Figure 7a. 
The computed activation energies are 2.5 and $1.9 \mathrm{kcal} \cdot \mathrm{mol}^{-1}$ for MINT-NDI and MINT-Pyr respectively, and 0.6 and $2.1 \mathrm{kcal} \cdot \mathrm{mol}^{-1}$ for MINT-AQ and MINT-exTTF. The order is MINT-NDI $\rightarrow$ MINT-exTTF $\rightarrow$ MINT-Pyr $\rightarrow$ MINT-AQ. As a check, the same quantities were also computed for MINT-NDI and MINT-Pyr at the xTB-GTFN level, yielding similar, yet lower barriers in both cases, and will not be discussed here. Note that these computed activation energies are an upper estimate for the translation of the macrocycles on the SWNT. Similar barriers are expected for pirouetting, as the motion will imply the concurrent combination of shuttling and pirouetting. This together with the flexibility of the macrocycles might lower these values.

The existence of these activation barriers depends on the fact that the motion of the macrocycle's aromatic moieties, namely benzene, anthracene and pyrene must pass through less favoured positions with respect to the SWNT. Geometrically, by analogy with graphite, these higher energy regions should be characterized by an increased number of atoms of these moieties lying atop of atoms of the underlying SWNT network. The comparison of the molecular structures of the maximum and minima energies for MINT-NDI longitudinal motion, see Figure 7b, illustrates this. At the maximum energy, both pyrenes show the largest overlap with the underlying carbon network, while at the minimum, a graphite-like $\mathrm{AB}$ pattern is observed in both cases. 


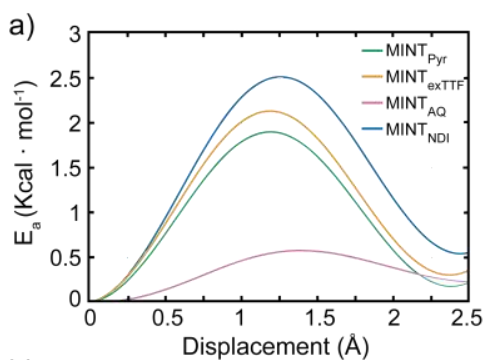

b)

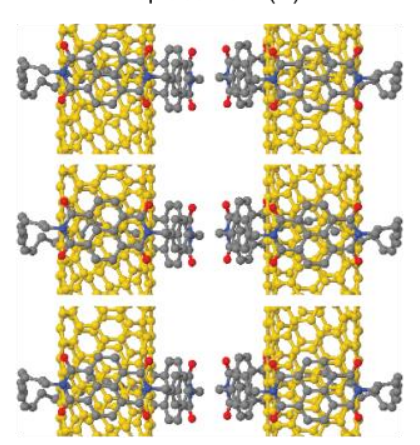

Figure 7. a) Activation energies for rigid displacement of the different macrocycles on top of the SWNT (DFTB-OB3). b) Chemical minimum (top), maximum (middle) and second minimum (bottom) for the rigid translation of MINT-NDI, the two orientations show the commensuration of the two pyrene moieties in the molecule.

The computed energetics indicate that, as observed in the molecular dynamics trajectories, at room temperature, the macrocycles should slide on the SWNT. Except for MINT-AQ, this movement might not be continuous but proceed through a series of tiny/fast jumps. For MINT-AQ, the barrier is too low and the macrocycle may glide freely. This finding fits remarkably well with the fact that molecular dynamics simulations of MINT-AQ showed enhanced mobility with respect to the other MINTs.

Moreover, we find that there is no significant correlation between $E_{a c t}$ or the area or number of short intermolecular contacts, and the motion observed in the MD simulations (see SI, S7). Instead, the presence/lack of a barrier and its height depends on the commensurability of each macrocycle aromatic moieties with the SWNT. By analogy with graphite, AA commensuration will be obtained when the atoms from the PAHS units sit atop of the atoms from the SWNT while AB commensuration will be obtained if the atoms from the PAHS sit near the center of the underlying hexagons. ${ }^{38}$ The minimum structures yield different patterns and orientations for the four molecules under study (see SI, S6). The commensurability of the aromatic moieties for MINT-AQ, whose barrier is considerably lower than in 
the other three cases is interesting. In this case, one of the anthracene moieties is sitting mostly on top of an anthracene section of the SWNT. In other words, the MINT-AQ minima is near AA registry. This explain the penalty in binding energy and the smoother and lower barrier. Furthermore, the MINT-NDI, which has the largest barrier shows the best AB commensuration. Lastly, MINT-exTTF and MINT-Pyr show similar lower barriers and only partial $\mathrm{AB}$ commensuration. This analysis reveals how the molecular structure and, specifically, the precise way each macrocycle wraps itself around the $(6,5)$ SWNT defines not only the binding but also the barriers and thus the shuttling and pirouetting dynamics.

In summary, we use MINTs as model systems to study movement of molecular fragments with respect to atomically flat surfaces. In particular, we have studied the sub-molecular motion of different organic macrocycles along and around SWNTs. MD simulations show that these movements do not depend on the interaction energy between the macrocycle and SWNT. Semiempirical quantum calculations yield very small energy barriers for motion, lower than rotation around a single $\mathrm{C}-\mathrm{C}$ bond in ethane. Globally, we conclude that the movement described by the different macrocycles is quasi barrier-less, that is, it responds to a zero-load, flat energy profile, in which the depth of the energy well does not affect motion. The slight differences between MINTs are better explained in terms of macrocycleSWNT conmensurability. These findings are in agreement with a direct atomic origin of Amontons' law, where no load implies no friction. Most likely, the same observation can be extended to molecular systems moving with respect to atomically flat surfaces, where there are no obvious energetic minima/maxima in the trajectory, in line with the "superlubricity" phenomenon described for graphene nanoribbons on gold surfaces. ${ }^{18}$

\section{ASSOCIATED CONTENT}

We have considered a chiral (6,5), single wall nanotube (SWNTs) of length $98.2 \AA$ and $7.75 \AA$ diameter containing 918 carbon atoms. DFT calculations were computed at the B97D/3-21G* level employing Gaussian suite of programs. ${ }^{39}$ MD simulations were performed using AMBER 12 software package with the AMBER12 force field ${ }^{40}$ supplemented by the general AMBER force field (GAFF). ${ }^{41}$ Analysis and 
visualization of MD trajectories were performed with VMD software. The barriers for the shuttling motion were computed at the semiempirical tight-binding DFTB-D3 level ${ }^{42}$ using the OB3 parameters set ${ }^{43}$ with the software DFTB.$+{ }^{44}$ A more detailed account of the models can be found in the SI.

\section{AUTHOR INFORMATION}

\section{Corresponding Authors}

Manuel Melle-Franco - CICECO - Aveiro Institute of Materials, Department of Chemistry, University of Aveiro, 3810-193 Aveiro, Portugal; Email: manuelmelle.research@gmail.com

Emilio M. Pérez - IMDEA Nanociencia, C/Faraday 9, Ciudad Universitaria de Cantoblanco, 28049 Madrid, Spain; Email: emilio.perez@imdea.org

\section{Authors}

Julia Villalva - IMDEA Nanociencia, C/Faraday 9, Ciudad Universitaria de Cantoblanco, 28049 Madrid, Spain

Belén Nieto-Ortega - IMDEA Nanociencia, C/Faraday 9, Ciudad Universitaria de Cantoblanco, 28049 Madrid, Spain

\section{ORCID}

Julia Villalva - 0000-0002-5628-2006

Belén Nieto-Ortega - 0000-0002-1834-3664

Manuel Melle-Franco - 0000-0003-1929-0477

Emilio M. Pérez - 0000-0002-8739-2777

\section{Notes}

The authors declare no competing financial interest.

\section{ACKNOWLEDGMENT}

We acknowledge funding from the European Union (ERC-StG-307609, ERC PoC 842606), MINECO (CTQ2014-60541-P and CTQ2017-86060-P), and the Comunidad de Madrid and the European Structural Funds for their financial support through FotoArt-CM project (S2018/NMT-4367) and MAD2D-CM S2013/MIT-3007. IMDEA Nanociencia acknowledges support from the "Severo Ochoa" 
Programme for Centres of Excellence in R\&D (MINECO, grant SEV-2016-0686). The computational work was supported by the Campus de Excelencia Internacional UAM+CSIC. MMF would like to acknowledge support from the Portuguese Foundation for Science and Technology (FCT), under the projects IF/00894/2015, and CICECO-Aveiro Institute of Materials, FCT Ref. UID/CTM/50011/2019, UIDB/50011/2020 \& UIDP/50011/2020, financed by national funds through the FCT/MEC and when appropriate co-financed by FEDER under the PT2020 Partnership Agreement. Additionally, we express our gratitude to the Supercomputing and Bioinnovation Center (SCBI) of the University of Málaga (Spain) for their support and resources.

\section{REFERENCES}

(1) Han, J.; Globus, A.; Jaffe, R.; Deardorff, G. Nanotechnology 1997, 8, 95.

(2) Stoddart, J. F. Chem. Soc. Rev. 2009, 38, 1802.

(3) Kay, E. R.; Leigh, D. A.; Zerbetto, F. Angewandte Chemie-International Edition 2007, 46, 72.

(4) Naranjo, T.; Lemishko, K. M.; Lorenzo, S. d.; Somoza, Á.; Ritort, F.; Pérez, E. M.; Ibarra, B. Nat. Commun. 2018.

(5) Meng, H.; Xue, M.; Xia, T.; Zhao, Y.-L.; Tamanoi, F.; Stoddart, J. F.; Zink, J. I.; Nel, A. E. J. Am. Chem. Soc. 2010, 132, 12690.

(6) Thomas, C. R.; Ferris, D. P.; Lee, J.-H.; Choi, E.; Cho, M. H.; Kim, E. S.; Stoddart, J. F.; Shin, J.-S.; Cheon, J.; Zink, J. I. J. Am. Chem. Soc. 2010, 132, 10623.

(7) Zhao, Y.-L.; Li, Z.; Kabehie, S.; Botros, Y. Y.; Stoddart, J. F.; Zink, J. I. J. Am. Chem. Soc. 2010, 132, 13016.

(8) Kohsaka, Y.; Koyama, Y.; Takata, T. Angew. Chem. Int. Ed. 2011, 50, 10417.

(9) Du, G.; Moulin, E.; Jouault, N.; Buhler, E.; Giuseppone, N. Angew. Chem. Int. Ed. 2012, 51, 12504.

(10) Meng, Z.; Xiang, J.-F.; Chen, C.-F. Chem. Sci. 2014, 5, 1520.

(11) Liu, L.; Liu, Y.; Liu, P.; Wu, J.; Guan, Y.; Hu, X.; Lin, C.; Yang, Y.; Sun, X.; Ma, J.; Wang, L. Chem. Sci. 2013, 4, 1701. 
(12) Xue, M.; Yang, Y.; Chi, X.; Yan, X.; Huang, F. Chem. Rev. 2015, 115, 7398.

(13) Mo, Y.; Turner, K. T.; Szlufarska, I. Nature 2009, 457, 1116.

(14) Gao, J.; Luedtke, W. D.; Gourdon, D.; Ruths, M.; Israelachvili, J. N.; Landman, U. J. Phys. Chem. B 2004, 108, 3410.

(15) Dienwiebel, M.; Verhoeven, G. S.; Pradeep, N.; Frenken, J. W. M.; Heimberg, J. A.; Zandbergen, H. W. Phys. Rev. Lett. 2004, 92, 126101.

(16) Guo, Y.; Guo, W.; Chen, C. Phys. Rev. B 2007, 76, 155429.

(17) Li, Q.; Lee, C.; Carpick, R. W.; Hone, J. Phys. Status Solidi B 2010, 247, 2909.

(18) Kawai, S.; Benassi, A.; Gnecco, E.; Söde, H.; Pawlak, R.; Feng, X.; Müllen, K.; Passerone, D.; Pignedoli, C. A.; Ruffieux, P.; Fasel, R.; Meyer, E. Science 2016, 351, 957.

(19) Vilhena, J. G.; Pimentel, C.; Pedraz, P.; Luo, F.; Serena, P. A.; Pina, C. M.; Gnecco, E.; Pérez, R. ACS Nano 2016, 10, 4288.

(20) Vazirisereshk, M. R.; Ye, H.; Ye, Z.; Otero-de-la-Roza, A.; Zhao, M.-Q.; Gao, Z.; Johnson, A. T. C.; Johnson, E. R.; Carpick, R. W.; Martini, A. Nano Lett. 2019, 19, 5496.

(21) Ouyang, W.; Mandelli, D.; Urbakh, M.; Hod, O. Nano Lett. 2018, 18, 6009.

(22) Li, S.; Li, Q.; Carpick, R. W.; Gumbsch, P.; Liu, X. Z.; Ding, X.; Sun, J.; Li, J. Nature 2016, $539,541$.

(23) Berman, A.; Drummond, C.; Israelachvili, J. Tribol. Lett. 1998, 4, 95.

(24) Mena-Hernando, S.; Perez, E. M. Chem. Soc. Rev. 2019, 48, 5016.

(25) Perez, E. M. Chem. Eur. J. 2017, 23, 12681.

(26) Juan, A. d.; Pouillon, Y.; Ruiz - González, L.; Torres - Pardo, A.; Casado, S.; Martín, N.; Rubio, Á.; Pérez, E. M. Angew. Chem. Int. Ed. 2014, 53, 5394.

(27) Lopez-Moreno, A.; Perez, E. M. Chem. Commun. 2015, 51, 5421.

(28) Martinez-Perinan, E.; de Juan, A.; Pouillon, Y.; Schierl, C.; Strauss, V.; Martin, N.; Rubio, A.; Guldi, D. M.; Lorenzo, E.; Perez, E. M. Nanoscale 2016, 8, 9254. 
Blazquez, J. P.; Vilatela, J. J.; Pisignano, D.; Perez, E. M. ACS Nano 2016, 10, 8012.

(31) Blanco, M.; Nieto-Ortega, B.; de, J. A.; Vera-Hidalgo, M.; Lopez-Moreno, A.; Casado, S.;

Perez, E. M.; Gonzalez, L. R.; Gonzalez-Calbet, J. M.; Sawada, H. Nat. Commun. 2018, 9, 2671.

(32) Song, W.; Pang, P.; He, J.; Lindsay, S. ACS Nano 2013, 7, 689.

(33) Ma, M. D.; Shen, L.; Sheridan, J.; Liu, J. Z.; Chen, C.; Zheng, Q. Phy. Rev. E 2011, 83, 036316.

(34) Verberck, B.; Cambedouzou, J.; Vliegenthart, G. A.; Gompper, G.; Launois, P. Carbon 2011, $49,2007$.

(35) Khlobystov, A. N.; Porfyrakis, K.; Kanai, M.; Britz, D. A.; Ardavan, A.; Shinohara, H.; Dennis, T. J. S.; Briggs, G. A. D. Angew. Chem. Int. Ed. 2004, 43, 1386.

(36) Liu, H.; He, J.; Tang, J.; Liu, H.; Pang, P.; Cao, D.; Krstic, P.; Joseph, S.; Lindsay, S.; Nuckolls, C. Science 2010, 327, 64 .

(37) Salvador, A. F.; Martins, G.; Melle-Franco, M.; Serpa, R.; Stams, A. J. M.; Cavaleiro, A. J.; Pereira, M. A.; Alves, M. M. Environ. Microbiol. 2017, 19, 2727.

(38) Strutyński, K.; Gomes, J. A. N. F.; Melle-Franco, M. J. Phys. Chem. A. 2014, 118, 9561.

(39) Frisch, M. J.; Trucks, G. W.; Schlegel, H. B.; Scuseria, G. E.; Robb, M. A.; Cheeseman, J. R.; Scalmani, G.; Barone, V.; Petersson, G. A.; Nakatsuji, H.; Li, X.; Caricato, M.; Marenich, A. V.; Bloino, J.; Janesko, B. G.; Gomperts, R.; Mennucci, B.; Hratchian, H. P.; Ortiz, J. V.; Izmaylov, A. F.; Sonnenberg, J. L.; Williams; Ding, F.; Lipparini, F.; Egidi, F.; Goings, J.; Peng, B.; Petrone, A.; Henderson, T.; Ranasinghe, D.; Zakrzewski, V. G.; Gao, J.; Rega, N.; Zheng, G.; Liang, W.; Hada, M.; Ehara, M.; Toyota, K.; Fukuda, R.; Hasegawa, J.; Ishida, M.; Nakajima, T.; Honda, Y.; Kitao, O.; Nakai, H.; Vreven, T.; Throssell, K.; Montgomery Jr., J. A.; Peralta, J. E.; Ogliaro, F.; Bearpark, M. J.; Heyd, J. J.; Brothers, E. N.; Kudin, K. N.; Staroverov, V. N.; Keith, T. A.; Kobayashi, R.; Normand, J.; Raghavachari, K.; Rendell, A. P.; Burant, J. C.; Iyengar, S. S.; Tomasi, J.; Cossi, M.; Millam, J. M.; 
Klene, M.; Adamo, C.; Cammi, R.; Ochterski, J. W.; Martin, R. L.; Morokuma, K.; Farkas, O.; Foresman, J. B.; Fox, D. J. Wallingford, CT, 2016.

(40) Hornak, V.; Abel, R.; Okur, A.; Strockbine, B.; Roitberg, A.; Simmerling, C. Proteins 2006, 65, 712.

(41) Wang, J.; Wolf, R. M.; Caldwell, J. W.; Kollman, P. A.; Case, D. A. J. Comput. Chem. 2004, 25, 1157.

(42) Kubillus, M.; Kubař, T.; Gaus, M.; Řezáč, J.; Elstner, M. J. Chem. Theory Comput. 2015, 11, 332.

(43) Gaus, M.; Goez, A.; Elstner, M. J. Chem. Theory Comput. 2013, 9, 338.

(44) Aradi, B.; Hourahine, B.; Frauenheim, T. J. Phys. Chem. A. 2007, 111, 5678.

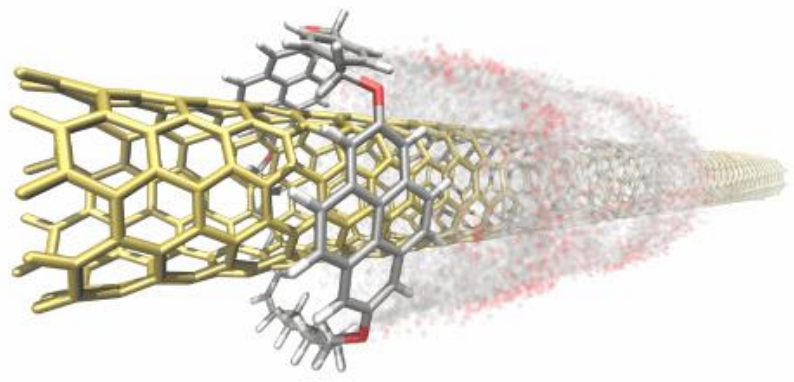

